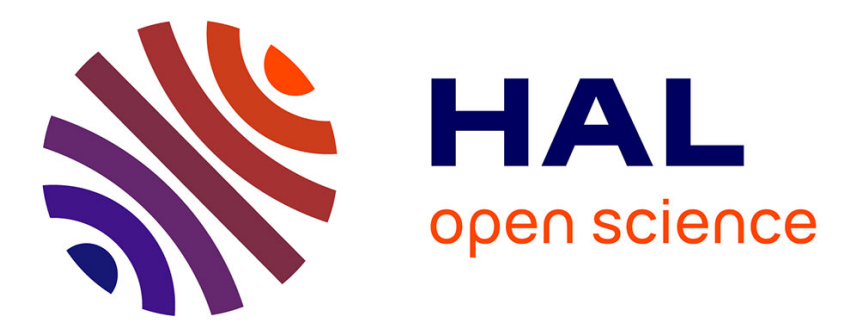

\title{
Blood flow in the cerebral venous system: modeling and simulation
}

Olivia Miraucourt, Stéphanie Salmon, Marcela Szopos, Marc Thiriet

\section{To cite this version:}

Olivia Miraucourt, Stéphanie Salmon, Marcela Szopos, Marc Thiriet. Blood flow in the cerebral venous system: modeling and simulation. Computer Methods in Biomechanics and Biomedical Engineering, 2016, 10.1080/10255842.2016.1247833 . hal-01384285

\section{HAL Id: hal-01384285 \\ https://hal.sorbonne-universite.fr/hal-01384285}

Submitted on 20 Oct 2016

HAL is a multi-disciplinary open access archive for the deposit and dissemination of scientific research documents, whether they are published or not. The documents may come from teaching and research institutions in France or abroad, or from public or private research centers.
L'archive ouverte pluridisciplinaire HAL, est destinée au dépôt et à la diffusion de documents scientifiques de niveau recherche, publiés ou non, émanant des établissements d'enseignement et de recherche français ou étrangers, des laboratoires publics ou privés. 
To appear in a journal

Vol. 00, No. 00, Month 20XX, 1-16

\title{
Blood flow in the cerebral venous system: modeling and simulation
}

\author{
Olivia Miraucourt ${ }^{\mathrm{a}}$, Stéphanie Salmon ${ }^{\mathrm{a} *}$, Marcela Szopos ${ }^{\mathrm{b}}$ and Marc Thiriet ${ }^{\mathrm{c}, \mathrm{d}}$ \\ ${ }^{a}$ Laboratoire de Mathématiques, EA 4535 FR CNRS ARC 3399, Université de Reims Champagne-Ardenne, \\ UFR Sciences Exactes et Naturelles, Moulin de la Housse - BP 1039, 51687 Reims CEDEX 2, France \\ ${ }^{b}$ Institut de Recherche Mathématique Avancée, UMR 7501 CNRS/Université de Strasbourg, 7 rue René \\ Descartes, 67084 Strasbourg CEDEX, France \\ ${ }^{c}$ Laboratoire J.-L. Lions, UMR 7598 CNRS/Université Pierre et Marie Curie-Paris 6, 75005 Paris, France \\ ${ }^{d}$ INRIA, Equipe-projet REO, 78153 Le Chesnay, France
}

(v4.0 released February 2014)

\begin{abstract}
The development of a software platform incorporating all aspects, from medical imaging data, through three-dimensional reconstruction and suitable meshing, up to simulation of blood flow in patient-specific geometries, is a crucial challenge in biomedical engineering. In the present study, a fully three-dimensional blood flow simulation is carried out through a complete rigid macrovascular circuit, namely the intracranial venous network, instead of a reduced order simulation and partial vascular network. The biomechanical modeling step is carefully analyzed and leads to the description of the flow governed by the dimensionless Navier-Stokes equations for an incompressible viscous fluid. The equations are then numerically solved with a free finite element software using five meshes of a realistic geometry obtained from medical images to prove the feasibility of the pipeline. Some features of the intracranial venous circuit in the supine position such as asymmetric behavior in merging regions are discussed.
\end{abstract}

Keywords: intracranial venous flow; computational fluid dynamics; Navier-Stokes equations; 3D reconstruction.

\section{Introduction}

The cerebral vasculature is a highly complicated three-dimensional network, the role of which is the maintenance of a proper blood drainage. The large cerebral arteries (e.g. carotid arteries, basilar trunk, and the circle of Willis) are the focus of multiple investigations (Formaggia et al. (2009) and references therein). In this context, blood flow computational models are relevant to various clinical applications, such as check-up and follow-up of aneurysms (Cebral et al. (2005); Shojima et al. (2014); Retarekar et al. (2015)) and control after stenting (Wang et al. (2011)) or coiling (Morales et al. (2013)). In contrast, the behavior of the venous cerebral system is rarely explored. This compartment has its own features, such as the lack of parallelism between arterial and venous circulations (Schaller (2004)) and asymmetrical and much more varying between-subject architecture and behavior compared to the arterial circuit (Schaller (2004); Stoquart-Elsankari et al. (2009)).

In parallel to technological improvements of medical imaging techniques (Ozsarlak et al. (2004); Miyazaki, Lee (2008)), computational fluid dynamics evolved with the development of new numerical schemes and high performance computing. However, despite recent progress in blood flow simulations, open problems still exist. They derive not only from computational challenges

${ }^{*}$ Corresponding author. Email: stephanie.salmon@univ-reims.fr 
and cost issues, but also from the difficulty to accurately model the multiple physiological assumptions of these complex multiphysics and multiscale phenomena. In this methodological framework, two strategies are generally considered.

The first strategy consists in carrying out reduced order simulations, i.e., to consider either zero-dimensional (0D) lumped-parameter models, exploiting the electric circuit analogy for the circulatory system, or one-dimensional (1D) distributed parameter models, thereby dealing with large vascular networks: Ursino, Lodi (1997) (0D model of intracranial hemodynamics), Gadda et al. (2015) (lumped parameter model of the cerebral venous outflow), Reymond et al. (2009) (1D model of the arterial network), Ho et al. (2013) (1D flow model built on an anatomical geometry of the cerebral venous system) and Müller, Toro (2014a), Müller, Toro (2014b) (closed-loop 1D model of the human circulation, with a special focus on the venous system).

Alternatively, three-dimensional (3D) computational fluid dynamics simulations can be carried out in patient-specific geometries. Classically, modeling is limited to relatively small samples of the vascular circuit, possibly coupled to $0 \mathrm{D}$ and $1 \mathrm{D}$ models, whether or not wall deformability is incorporated in modeling (Formaggia et al. (2009); Cebral et al. (2005); Boissonnat et al. (2005)). Recent attempts were proposed for the cerebral arterial network: Passerini et al. (2009) (1D description of the circle of Willis, coupled to a fully 3D model of a carotid artery), Xiao et al. (2013) (3D/0D computational model of hemodynamics within a deformable full-body arterial network), and venous system: Caiazzo et al. (2015) (3D description of blood flow in rigid jugular veins coupled with a 1D model for major intracranial veins).

Our contribution is to rely on 3D blood flow modeling in more complete vascular networks, in order to better describe blood flow patterns in complicated geometries. In particular, such approach is relevant in the cerebral venous network whose geometry significantly differs from arteries. The present work is aimed at proposing a complete computational modeling approach from medical image processing to numerical resolution. The study relies on developing and using open-source softwares, which enable a full handling of the underlying methods and afford guarantees in terms of availability as well as reproducibility. We focus on intracranial venous blood flow in supine position, that is, a drainage through the jugular veins, which are nevertheless not included in the present model, as they are strongly deformable. In particular, the present model assumes that intracranial veins located between the skull and the brain are rigid. The venous circuit is isolated, as reduced modeling approaches (e.g., $0 \mathrm{D}$ and 1D models) that can be coupled to 3D models need patient-specific geometrical and mechanical data that cannot be easily assessed.

The remainder of this article is structured as follows. Section 2 is devoted to the description of the venous network, Section 3 to the biomechanical model and its associated equation set, and Section 4 to the numerical procedure. Results and discussion are presented in Sections 5 and 6 . Concluding remarks and future prospects are gathered in the final section.

\section{Anatomical model of the large cerebral veins}

The large cerebral veins are localized in the leptomeninges and drain blood to the internal jugular veins that ensure the extracranial drainage of the brain perfusion. The realistic three-dimensional description of the geometry of the network was constructed starting from 3D angiographic images obtained by magnetic resonance angiography (MRA). More details on the different steps of the process can be found in Miraucourt et al. (2014).

The brain venous network is composed by input veins (Fig. 1): vein of Galen (7); internal cerebral vein (8); basilar vein (9); superior cerebral veins (10); superior anastomotic veins (11); 


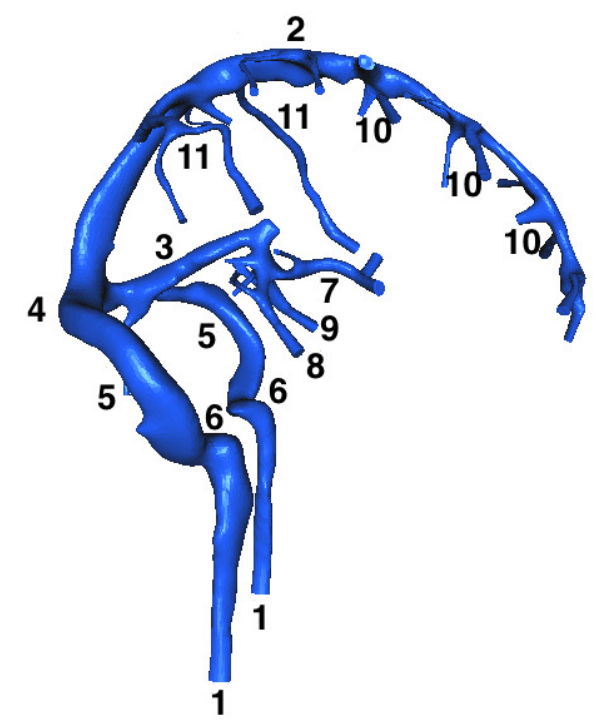

Figure 1. Cerebral venous network. 1: virtual exit veins; 2: superior sagittal sinus; 3: straight sinus; 4: confluence of sinuses; 5: lateral sinus (transverse portion); 6: lateral sinus (sigmoid portion); 7: vein of Galen; 8: internal cerebral vein; 9: basal vein; 10: superior cerebral veins; 11: superior anastomotic veins.

draining the blood into the superior sagittal sinus (2) and straight sinus (3), down to their confluence (4). The blood then passes into the transverse (5) and sigmoid parts (6) of the lateral sinuses, and reaches their corresponding extracranial regions.

Depending on the subject's position (i.e., lying vs. standing), the cerebral blood flows back to the cardiac pump using different set of cerebral veins (Gisolf et al. (2004); Stoquart-Elsankari et al. (2009)). In the supine position, the functional drainage from the left and right side of the venous circuit encompass large jugular veins that can be included in the $3 \mathrm{D}$ reconstruction. In the standing position, a collection of small veins drain the cerebral blood. However, current medical image acquisition processes do not include a more sophisticated description of the collateral pathways (the vertebral plexus and anastomoses leading blood to the downstream segments of the jugular veins) that were very recently shown to play an important role as an alternative route for venous outflow according to the subject position, that is, standing or sitting (Gadda et al. (2015)).

Neither the jugular veins, which drain the intracranial venous blood in the supine position, nor the collection of small veins, which drain the cerebral blood in the standing position, are included in the present model. However, two fictitious exit veins (Fig. 1 (1)) have been added to the computational domain, so that the outlet boundary condition does not affect the flow dynamics within the computational domain. Moreover, the downstream influence of a 3D unsteady flow can be physically mimicked. It is worth mentioning that in the considered venous network, valves are absent (Kiliç, Akakin (2008)), at the difference of the venous lower limb system, where a complex system of valves ensures the passage of blood upward from the feet against gravity.

Approximate values of vein radii, computed from the geometric vessel modeling process are compared with literature data in Table 1, with an emphasis on values previously used in 1D models of venous blood flow. These results are in good accordance, considering the individual variations. 
Table 1. Venous geometry: approximate magnitude $(\mathrm{mm})$ of the radius at some typical sites.

\begin{tabular}{|c|c|c|c|}
\hline \multirow{2}{*}{$\begin{array}{l}\text { Vessel name } \\
\text { Right internal jugular vein }\end{array}$} & \multicolumn{2}{|c|}{ Radius (in the literature) } & \multirow{2}{*}{$\begin{array}{l}\text { Radius (MRA, present study) } \\
2.59\end{array}$} \\
\hline & $\begin{array}{l}3.0 \rightarrow 7.0 \\
2.52 \rightarrow 3.99\end{array}$ & $\begin{array}{l}\text { Ho et al. (2013) } \\
\text { Müller, Toro (2014a) }\end{array}$ & \\
\hline Left internal jugular vein & $\begin{array}{l}3.0 \rightarrow 7.0 \\
3.99 \rightarrow 6.18\end{array}$ & $\begin{array}{l}\text { Ho et al. (2013) } \\
\text { Müller, Toro (2014a) }\end{array}$ & 2.34 \\
\hline Superior sagittal sinus & $\begin{array}{l}2.3 \rightarrow 3.0 \\
2.0 \rightarrow 3.19\end{array}$ & $\begin{array}{l}\text { Ho et al. (2013) } \\
\text { Müller, Toro (2014a) }\end{array}$ & $2.1 \rightarrow 4.9$ \\
\hline Straight sinus & $\begin{array}{l}1.8 \\
2.5\end{array}$ & $\begin{array}{l}\text { Ho et al. (2013) } \\
\text { Müller, Toro (2014a) }\end{array}$ & 3.3 \\
\hline Right transverse lateral sinus & $\begin{array}{l}4 \rightarrow 5 \\
1.78 \rightarrow 2.52\end{array}$ & $\begin{array}{l}\text { Schaller (2004) } \\
\text { Müller, Toro (2014a) }\end{array}$ & 2.9 \\
\hline Left transverse lateral sinus & $\begin{array}{l}4 \rightarrow 5 \\
3.09 \rightarrow 4.37\end{array}$ & $\begin{array}{l}\text { Schaller (2004) } \\
\text { Müller, Toro (2014a) }\end{array}$ & 4.9 \\
\hline Right sigmoid sinus & 2.52 & Müller, Toro (2014a) & 3.8 \\
\hline Left sigmoid sinus & $3.57 \rightarrow 3.99$ & Müller, Toro (2014a) & 5.2 \\
\hline Vein of Galen & $3.09 \rightarrow 4.0$ & Müller, Toro (2014a) & $2.06 \rightarrow 2.50$ \\
\hline Internal cerebral vein & 1.26 & Müller, Toro (2014a) & 2.07 \\
\hline Basal vein & 1.26 & Müller, Toro (2014a) & 2.03 \\
\hline Superior cerebral veins & - & & $1.55 \rightarrow 1.83$ \\
\hline Superior anastomotic veins & 1.0 & Müller, Toro (2014a) & $1.07 \rightarrow 1.86$ \\
\hline
\end{tabular}

\section{Biomechanical model}

The following standard blood properties and flow features and assumptions are used: (1) blood is incompressible; (2) blood is homogeneous, as the size of red blood cells (97\% of the flowing cell population) is much lower than the vein caliber; (3) the endothelial wetted surface is smooth at this length scale (the size of indentations linked to endothelial cell nuclei is of the same order of magnitude than of that of blood cells); (4) blood is Newtonian; (5) venous walls are rigid; (6) flow is isothermal; and (7) body forces are neglected (gravity and electromagnetic field as flow is computed at a time immediately after scanning, before standing up).

Rheology of blood and vascular walls still remain important modeling issues and challenging numerical tasks, that are not yet fully handled. The effect of stress history and blood thixotropy is, in general, not incorporated in blood flow models of the literature, thereby not representing properly blood rheology. Wall mechanics is represented, in general, by a simple shell model. In the absence of blood stagnation in the large compartment of the intracranial venous circuit (Table 1), that is, in the absence of red blood cell rouleau formation, blood can be supposed to remain Newtonian (Formaggia et al. (2009); Thiriet (2011)). Hence, thixotropic, viscoelastic, and shear-thinning features are omitted.

Intracranial veins run through the meninges, a quasi-incompressible biological tissue composed mostly of water, which are quite constrained between the quasi-incompressible brain and rigid skull. They are thus not susceptible to experience large deformations at normal transmural pressures. Moreover, recent computational sensitivity analyses (Sforza et al. (2010)) showed that neglecting wall motion is an acceptable hypothesis when studying intracranial arterial blood flow, although arteries are subjected to stronger changes in internal pressure. Wall rigidity assumption is more questionable for the jugular veins that drain the cerebral blood in the supine position, as these veins travel through the neck and are thus exposed to the ambient pressure and deformation engendered by pressure waves running along the carotid arteries (Section 6). In order to ensure wall rigidity hypothesis, the jugular veins are not included in the present model. 


\section{Dimensional analysis}

As a consequence of all these assumptions, we consider in the following the governing equations of an unsteady isothermal flow of a Newtonian incompressible fluid (mass density $\rho$, dynamic viscosity $\mu$, and kinematic viscosity $\nu=\mu / \rho)$ in an anatomical vessel conveyed with a velocity $\mathbf{u}(\mathbf{x}, t)(\mathbf{x}$ : Eulerian position, $t$ : time), derived from the mass and momentum conservation. They are known as the non-linear Navier-Stokes equations

$$
\begin{gathered}
\rho\left(\partial_{t}+\mathbf{u} \cdot \boldsymbol{\nabla}\right) \mathbf{u}=\mathbf{f}+\boldsymbol{\nabla} \cdot \mathbf{C} \\
\boldsymbol{\nabla} \cdot \mathbf{u}=0
\end{gathered}
$$

where $\mathbf{C}=-p \mathbf{I}+2 \mu \mathbf{D}$ is the stress tensor, $p$ is the pressure, $\mathbf{I}$ the metric tensor and $\mathbf{D}=\frac{1}{2}\left(\boldsymbol{\nabla} \mathbf{u}+(\boldsymbol{\nabla} \mathbf{u})^{T}\right)$ is the rate of deformation tensor for a Newtonian fluid. In isothermal conditions, Equations (1) lead to the simplified form of the Navier-Stokes equations in the absence of body forces $\mathbf{f}$, namely

$$
\rho\left(\partial_{t} \mathbf{u}+(\mathbf{u} \cdot \boldsymbol{\nabla}) \mathbf{u}\right)=-\nabla p+\mu \Delta \mathbf{u}
$$

The Navier-Stokes equations can be transformed into a dimensionless form, using appropriate scales for the length $\left(L^{\star}\right.$; either the boundary layer thickness or the duct hydraulic radius for fullydeveloped flow $)$, time $\left(T^{\star}\right.$; either the flow cycle period $\left[T^{\star} \equiv T\right]$ or the inverse of the flow pulsation $\left.\left[T^{\star} \propto \omega^{-1}\right]\right)$, velocity $\left(U^{\star}\right.$; either the mean or maximal cross-sectional velocity), and the pressure $\left(P^{\star}\right)$. Therefore, we have

$$
\tilde{t}=t / T^{\star}, \quad \tilde{x}=x / L^{\star}, \quad \tilde{u}=u / U^{\star}, \quad \tilde{p}=p / P^{\star}
$$

and the dimensionless differential operators are then expressed by $\widetilde{\nabla}=L^{\star} \nabla$ and $\widetilde{\boldsymbol{\Delta}}=L^{\star^{2}} \boldsymbol{\Delta}$.

The classical formulation of the dimensionless Navier-Stokes equations are hence obtained

$$
\frac{\rho U^{\star}}{T^{\star}} \partial_{\tilde{t}} \tilde{\mathbf{u}}+\frac{\rho U^{\star^{2}}}{L^{\star}}(\tilde{\mathbf{u}} \cdot \tilde{\nabla}) \tilde{\mathbf{u}}=-\frac{P^{\star}}{L^{\star}} \widetilde{\nabla} \tilde{p}+\frac{\mu U^{\star}}{L^{\star^{2}}} \widetilde{\Delta} \tilde{\mathbf{u}}
$$

According to the predominant force type, either convective inertial forces or viscous forces, the dimensionless coefficients can be expressed differently. In steady flows, the pressure gradient balances the friction (and gravity when it has significant effect, as in blood vessels in standing position). In quasi-steady flows, the rate of change in boundary conditions are so slow that the momentum has time to diffuse during the flow period. In unsteady flows, the local inertia effects can be preponderant.

Since we are interested in the case when convective inertia is predominant, the dimensionless Navier-Stokes equations become (when tilde notation is omitted for the sake of simplicity)

$$
\text { St } \partial_{t} \mathbf{u}+(\mathbf{u} \cdot \nabla) \mathbf{u}=-\nabla p+\operatorname{Re}^{-1} \boldsymbol{\Delta} \mathbf{u}
$$

where $\operatorname{Re}=\frac{\rho L^{*} V^{*}}{\mu}, \mathrm{St}=\frac{\omega L^{*}}{V *}=\frac{\mathrm{Sto}^{2}}{\operatorname{Re}}$ and $\mathrm{Sto}=L^{*} \sqrt{\frac{\omega}{\nu}}$ are the Reynolds number, Strouhal number and Stokes number, respectively.

Data used in the present model to compute the flow-governing dimensionless parameters are given in Table 2. However, patient-specific blood flow data are usually not collected from routine 
Table 2. Values of the mechanical parameters in the model.

\begin{tabular}{lllll}
\hline Parameter & Value & Unit & Source & \\
\hline Mass density & $\rho$ & 1055 & {$\left[\frac{\mathrm{kg}}{\mathrm{m}^{3}}\right]$} & Thiriet (2008) \\
\hline Dynamic viscosity & $\mu$ & $3.5 \cdot 10^{-3}$ & {$[P a \cdot s]$} & Thiriet (2008) \\
\hline Kinematic viscosity & $\nu$ & $(2.8-3.8) \cdot 10^{-6}$ & {$\left[\frac{\mathrm{m}^{2}}{\mathrm{~s}}\right]$} & Thiriet (2008) \\
\hline Flow pulsation & $\omega$ & 1 & {$\left[\frac{1}{\mathrm{~s}}\right]$} & Thiriet (2008) \\
\hline $\begin{array}{l}\text { Cross-sectional velocity } \\
\text { (clinical studies, jugular vein) }\end{array}$ & $V^{*}$ & $\begin{array}{l}(10-11) \cdot 10^{-2} \\
(8.5-11.3) \cdot 10^{-2}\end{array}$ & {$\left[\frac{\mathrm{m}}{\mathrm{s}}\right]$} & $\begin{array}{l}\text { Ho et al. (2013) } \\
\text { Ogoh et al. (2011) } \\
\text { Schaller (2004) }\end{array}$ \\
\hline $\begin{array}{l}\text { Cross-sectional velocity (clinical } \\
\text { studies, superior sagittal sinus) }\end{array}$ & $V^{*}$ & $15 \cdot 10^{-2}$ & {$\left[\frac{\mathrm{m}}{\mathrm{s}}\right]$} & Schaller (2004) \\
& & $(15.2 \pm 3) \cdot 10^{-2}$ & {$\left[\frac{\mathrm{m}}{\mathrm{s}}\right]$} & Gideon et al. (1996) \\
\hline
\end{tabular}

Table 3. Values of the dimensionless governing parameters.

\begin{tabular}{llll}
\hline Vessel name & Reynolds number & Stokes number & Strouhal number \\
\hline Internal jugular vein & $75 \rightarrow 1055$ & $1.38 \rightarrow 3.84$ & $0.014 \rightarrow 0.030$ \\
\hline Superior sagittal sinus & $90 \rightarrow 144$ & $1.10 \rightarrow 1.75$ & $0.013 \rightarrow 0.021$ \\
\hline
\end{tabular}

clinical examinations. Hence, scarce literature data are used for the velocity magnitude in cerebral veins. Computed values for the Reynolds, Stokes, and Strouhal number are reported in Table 3. The order of magnitude of the Reynolds number shows the importance of the convective forces, whereas the values of the Stokes and Strouhal numbers are important to assess the flow unsteadiness. The flow in the cerebral veins is thus governed by the Navier-Stokes equations for an incompressible viscous fluid, in a quasi-steady regime.

\section{Initial and boundary conditions}

The initial status of the fluid velocity, $\mathbf{u}_{\mathbf{0}}$ has to be carefully prescribed, since it has to be divergence-free to be admissible. Unfortunately, in hemodynamic computations, $\mathbf{u}_{\mathbf{0}}$ is usually unknown, hence chosen equal to zero everywhere or, as a better guess, as the solution of a stationary Stokes problem.

The inflow is situated at the microcirculation exit, far from the thorax, hence far from the effects of breathing and cardiac pumping (Thiriet (2008)). Since flow is not fully developed, both Poiseuille (steady case) or Womersley (unsteady case) flows are not appropriate. An impulsive start, with a uniform injection velocity is then employed. However, rigorous analysis of the unsteady Navier-Stokes equations demands regularity of the flow solution down to $t=0$, so this condition must be modeled as smoothly as possible. In practice, a time-dependent multiplier of the form $\left(1-e^{-k t}\right)$, with $k$ constant, is introduced to ensure that the inflow data smoothly ramps up from zero to the desired steady state boundary condition (a constant function $\mathbf{u}_{\text {in }}$ ) as $t \rightarrow \infty$. This velocity inlet condition is imposed at the 29 entrance sections of the mesh. The value of $\mathbf{u}_{i n}$ is chosen in accordance to Table 2 and the parameter $k$ is chosen such that the steady state value is reached at the third of the first cycle.

At the level of the exit veins, in a first approximation, we use a traction-free boundary condition and focus on the intracranial hemodynamics to minimize the influence of the outflow section. For more realistic boundary conditions, we report to another work carried out in the VIVABRAIN 
project $^{1}$ (Chabannes et al. (2015)).

In summary, the imposed boundary conditions are the following: (i) a smoothed impulsive velocity profile at entries; (ii) homogeneous natural conditions at exits; and (iii) no-slip condition at walls assumed to be rigid.

\section{Final model}

The mathematical model that we consider in the sequel is the Navier-Stokes equations with mixed boundary conditions:

$$
\left\{\begin{aligned}
\partial_{t} \mathbf{u}+\mathbf{u} \cdot \nabla \mathbf{u}-\nu \Delta \mathbf{u}+\boldsymbol{\nabla} p & =\mathbf{f} & & \text { in } \Omega \times(0, T) \\
\boldsymbol{\nabla} \cdot \mathbf{u} & =0 & & \text { in } \Omega \times(0, T) \\
\mathbf{u} & =\mathbf{g}_{1} & & \text { on } \Gamma_{D} \times(0, T) \\
\nu \frac{\partial \mathbf{u}}{\partial \mathbf{n}}-p \mathbf{n} & =\mathbf{g}_{2} & & \text { on } \Gamma_{N} \times(0, T) \\
\left.\mathbf{u}\right|_{t=0} & =\mathbf{u}_{0} & & \text { in } \Omega
\end{aligned}\right.
$$

where $\Omega \subset \mathbb{R}^{3}$ is a bounded domain with a Lipschitz-continuous boundary $\Gamma,(0, T)$ is a finite time interval. We recall that $\mathbf{u}=\left(u_{1}, u_{2}, u_{3}\right)$ is the fluid velocity, $p$ its pressure and $\nu$ its kinematic viscosity. We suppose that $\Gamma$ consists of two measurable parts: $\Gamma_{D}$, where Dirichlet boundary conditions are imposed, and $\Gamma_{N}$, where Neumann boundary conditions are prescribed. We denote by $\mathbf{n}$ the unit outward normal vector to $\Omega$ on its boundary $\Gamma$. The Dirichlet boundary conditions correspond in the non-homogeneous case to a velocity $\mathbf{g}_{1}$, generally prescribed at the inlets, and in the homogeneous case to the non-slip boundary condition prescribed at the vessel wall; the function $\mathbf{g}_{2}$ corresponds to the normal component of the stress tensor, prescribed at the outlets, which in the current model is set to zero.

\section{Numerical method}

As derived in the previous section, the equations under consideration are the unsteady incompressible Navier-Stokes equations (2), endowed with appropriate initial and boundary conditions. In this section, we describe the numerical approach we use in the sequel to compute an approximate solution to these equations, involving a time-scheme based on the characteristics method and a spatial discretization of finite element type. This algorithm is quite classical and is used as a reference to prove feasibility of the pipeline.

The main idea of the time discretization scheme is to take into account the fact that the terms $\partial_{t} \mathbf{u}+\mathbf{u} . \nabla \mathbf{u}$ can be written as the total derivative of $\mathbf{u}$ in the direction of the flow $\mathbf{u}$ (Pironneau (1982); Formaggia et al. (2009)). More precisely, let $\mathbf{x} \in \Omega$ and $t \in[0, T]$; we start by defining the characteristics $\mathcal{X}=\mathcal{X}(\tau ; t, \mathbf{x})$ associated to the vector field $\mathbf{u}$ as the solution to

$$
\left\{\begin{aligned}
\frac{\mathrm{d}}{\mathrm{d} \tau}[\mathcal{X}(\tau ; t, \mathbf{x})] & =\mathbf{u}(\mathcal{X}(\tau ; t, \mathbf{x}), t), \quad \tau \in(0, T) \\
\mathcal{X}(t ; t, \mathbf{x}) & =\mathbf{x}
\end{aligned}\right.
$$

The total derivative can then be written as:

$$
\left(\partial_{t} \mathbf{u}+\mathbf{u} . \nabla \mathbf{u}\right)(\mathbf{x}, t)=\left.\frac{\mathrm{d}}{\mathrm{d} \tau}[\mathbf{u}(\mathcal{X}(\tau ; t, \mathbf{x}), t)]\right|_{\tau=t}
$$

\footnotetext{
${ }^{1}$ www.vivabrain.fr
} 
where $\mathcal{X}(\tau ; t, \mathbf{x})$ is the position at time $\tau$ of a particle which has the position $\mathbf{x}$ at time $t$. Consequently, it can be approximated by:

$$
\left(\partial_{t} \mathbf{u}+\mathbf{u} \cdot \nabla \mathbf{u}\right)\left(\mathbf{x}, t^{n+1}\right)=\frac{\mathbf{u}\left(\mathbf{x}, t^{n+1}\right)-\mathbf{u}\left(\mathcal{X}^{n}(\mathbf{x}), t^{n}\right)}{\Delta t}+\mathcal{O}(\Delta t)
$$

where $\mathcal{X}^{n}(\mathbf{x})=\mathbf{x}-\mathbf{u}\left(\mathbf{x}, t^{n}\right) \Delta t+\mathcal{O}\left(\Delta t^{2}\right)$ is an approximation of $\mathcal{X}\left(t^{n+1} ; t^{n}, \mathbf{x}\right), \Delta t>0$ is the time-step and $t^{n}=n \Delta t, n \in \mathbb{N}$. As a consequence, the non-linear term is treated explicitly. The term containing $\mathcal{X}^{n}(\mathbf{x})$ goes to the right hand side, which yields a generalized Stokes problem, that can be solved as a standard Stokes problem, and can be handled numerically as such, with a preconditioned Uzawa/conjugate gradient algorithm.

In order to present the space discretization method, we introduce the functional spaces for the velocity $V=\left\{\mathbf{v} \in H^{1}(\Omega) \mid \mathbf{v}_{\left.\right|_{\Gamma_{D}}}=0\right\}$ and for the pressure $Q=L^{2}(\Omega)$, respectively. Let $\mathcal{T}$ be a triangulation of the domain $\Omega$ and $h$ the maximum of the diameters of the triangles of $\mathcal{T}$. We introduce the finite-dimensional spaces $V_{h}$ and $Q_{h}$ which are respectively contained in $V$ and $Q$

$$
\begin{aligned}
V_{h} & =\left\{\mathbf{v}_{h} \in \mathcal{C}^{0}(\bar{\Omega})^{d}, \forall K \in \mathcal{T}, \mathbf{v}_{h \mid K} \in \mathbb{P}_{2}(K)\right\} \\
Q_{h} & =\left\{q_{h} \in \mathcal{C}^{0}(\bar{\Omega}), \forall K \in \mathcal{T}, q_{h \mid K} \in \mathbb{P}_{1}(K)\right\}
\end{aligned}
$$

where $\mathbb{P}_{1}(K)$ (resp. $\mathbb{P}_{2}(K)$ ) is the space of polynomials of degree less than or equal to 1 (resp. 2 ) in each triangle of $\mathcal{T}$. The discretization spaces are chosen compatible - in the sense of the so-called inf-sup condition - to cope with the divergence constraint (Formaggia et al. 2009, Chapter 15).

The fully discrete problem then reads: find $\mathbf{u}_{h} \in V_{h}$ and $p_{h} \in Q_{h}$ such that

$$
\left\{\begin{aligned}
\frac{1}{\Delta t}\left(\mathbf{u}_{h}^{n+1}, \mathbf{v}_{h}\right)+\nu\left(\nabla \mathbf{u}_{h}^{n+1}, \nabla \mathbf{v}_{h}\right)-\left(p_{h}^{n+1}, \boldsymbol{\nabla} \cdot \mathbf{v}_{\mathbf{h}}\right) & =\left(\mathbf{f}_{h}^{n+1}, \mathbf{v}_{h}\right)+\frac{1}{\Delta t}\left(\mathbf{u}_{h}^{n} \circ X^{n}, \mathbf{v}_{h}\right) \\
& +\left(\mathbf{g}_{2 h}^{n+1}, \mathbf{v}_{h}\right)_{\Gamma_{N}} \\
\left(\boldsymbol{\nabla} \cdot \mathbf{u}_{h}^{n+1}, q_{h}\right) & =0
\end{aligned}\right.
$$

with $\mathbf{u}_{h}^{n+1}=\mathbf{g}_{1 h}^{n+1}$ on $\Gamma_{D}$.

Theoretically expected convergence in space for velocity error is of order 3 for the $L^{2}$-norm and of order 2 for the semi-norm $H^{1}$; for the pressure error, it is of order 2 for the $L^{2}$-norm and of order 1 for the semi-norm $H^{1}$ (Girault, Raviart (1986), Chapter 2). The characteristics method is proven to be unconditionally stable and expected to be of order 1 in time (Formaggia et al. 2009, Chapter 15).

The numerical solving approach relies on the finite element library FrEEFEM $++^{2}$ (Hecht (2012)) as described in (Miraucourt, Garnotel (2014)). To test the whole chain from images to computation, we developed an in-house software, based on the VisuALISATION ToolKiT ${ }^{3}$ in the framework of the VIVABRAIN project. Several meshes are obtained from the 3D reconstruction (Table 4).

We used the algorithm to compute flow with a time-step equal to $10^{-2} \mathrm{~s}$, thus providing a simulation of 2 seconds of a quasi-steady blood flow in the cerebral venous network. The CPU time with respect to the different meshes are summarized in Table 5. Finally, pressure and velocity

\footnotetext{
${ }^{2}$ http://www.freefem.org

${ }^{3} h t t p: / / w w w . v t k . o r g$
} 
Table 4. Mesh characteristics

\begin{tabular}{llllll}
\hline & Mesh 1 & Mesh 2 & Mesh 3 & Mesh 4 & Mesh 5 \\
\hline Number of elements & 66,885 & 94,690 & 151,634 & 212,679 & 535,453 \\
\hline Degrees of freedom & 405,663 & 552,126 & 884,469 & $1,148,127$ & $2,692,798$ \\
\hline Minimum Element size $(\mathrm{mm})$ & 0.10 & 0.12 & 0.08 & 0.07 & 0.05 \\
\hline Maximum Element size $(\mathrm{mm})$ & 7.02 & 6.70 & 5.60 & 4.72 & 3.85 \\
\hline
\end{tabular}

field computed on a finite-element basis are visualized with color-coded magnitudes according to PARAVIEW SOFTWARE ${ }^{4}$ and flow rates are calculated across selected cross-sections.

Table 5. CPU Time for the different meshes

\begin{tabular}{llllll}
\hline & Mesh 1 & Mesh 2 & Mesh 3 & Mesh 4 & Mesh 5 \\
\hline CPU time (s) & 3,447 & 4,853 & 8,386 & 13,747 & 43,122 \\
\hline
\end{tabular}

\section{Results}

We present in Fig. 2 the velocity field in the whole intracranial cerebral venous network. A complicated three-dimensional flow behavior is observed, especially in merging regions of the intracranial venous circuit. This asymmetric behavior appears for instance in a recirculation in the entrance segment of the right transverse sinus. The asymmetric feature of the venous flow can be explained, at least partly, by the asymmetric architecture of the venous network. The more refined the mesh, the clearer the recirculation.

Note that for comparison purposes, only MRI measurements in the supine position are extracted from the literature (which deals with geometrically reduced 1D models). In the present model, the predicted values of the velocity magnitude in the superior sagittal sinus range from $12.5 \cdot 10^{-2} \mathrm{~m} / \mathrm{s}$ to $18 \cdot 10^{-2} \mathrm{~m} / \mathrm{s}$, consistently with clinical data, that is, $15 \cdot 10^{-2} \mathrm{~m} / \mathrm{s}$ (Schaller (2004)) and $15.2 \pm$ $3 \cdot 10^{-2} \mathrm{~m} / \mathrm{s}$ (Gideon et al. (1996)) from a set of 14 control subjects using MR velocimetry. Flow rates calculated at inlets and outlets are identical $\left(\sim 8 \mathrm{ml} / \mathrm{s}\right.$ for an entry velocity $\mathbf{u}_{i n}=50 \mathrm{~mm} / \mathrm{s}$, corresponding to a physiological value (Stoquart-Elsankari et al. (2009)), hence guaranteeing mass conservation. Similar or slightly higher values, between $10 \mathrm{ml} / \mathrm{s}$ and $14 \mathrm{ml} / \mathrm{s}$ for the outflow were reported in the literature (Müller, Toro $(2014 a, b)$ ). Comparisons between the model predictions of flow rate values at selected cross-sections with MRI measurements reported in the literature are presented in Fig. 3.

\section{Discussion}

\subsection{Reduced dimensions models}

Previous studies mainly focused on the arterial compartment or investigated the venous intracranial dynamics by means of reduced 0D or 1D models. However, local values of vascular resistances and compliances remain, in general, unknown in the context of personalized studies. Moreover, they cannot yield detailed flow patterns. In addition, it has been recently demonstrated in Xiao et al. (2014) that a satisfactory agreement is obtained by 1D models during decelerating phase, but significant differences appear during accelerating phase, partly explained by the inability of

\footnotetext{
${ }^{4}$ http://www.paraview.org
} 

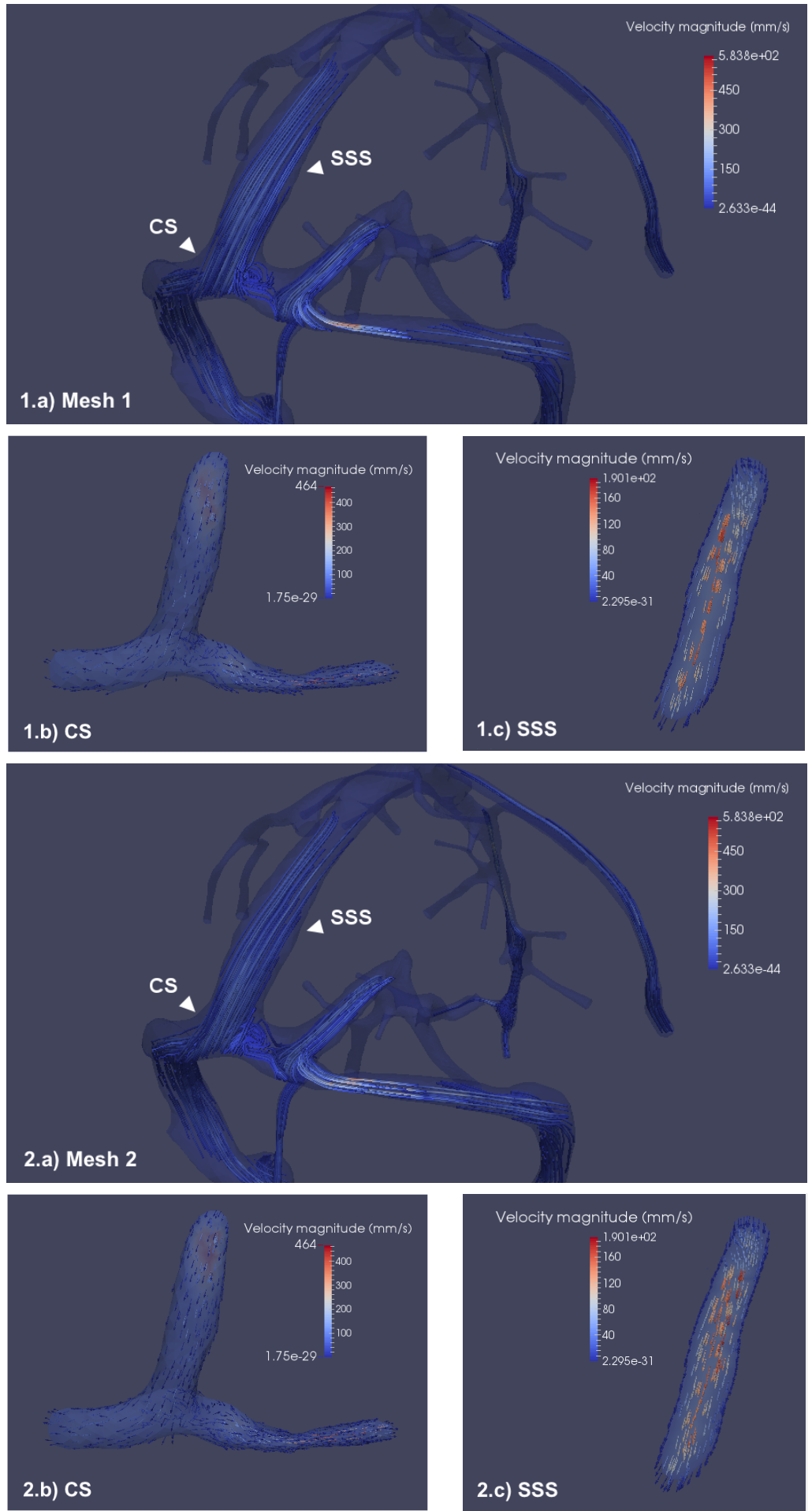

Velocity magnitude $(\mathrm{mm} / \mathrm{s})$
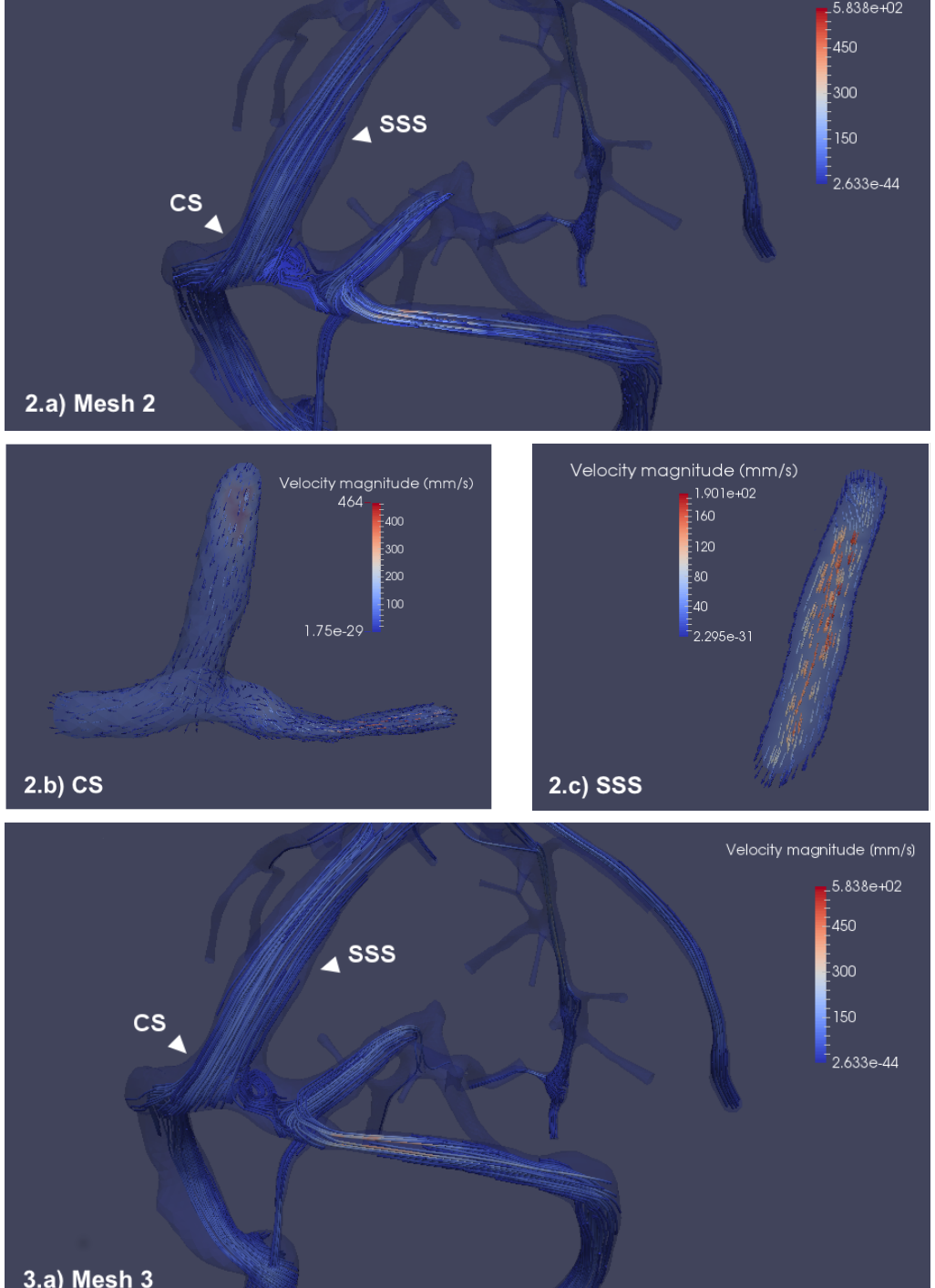

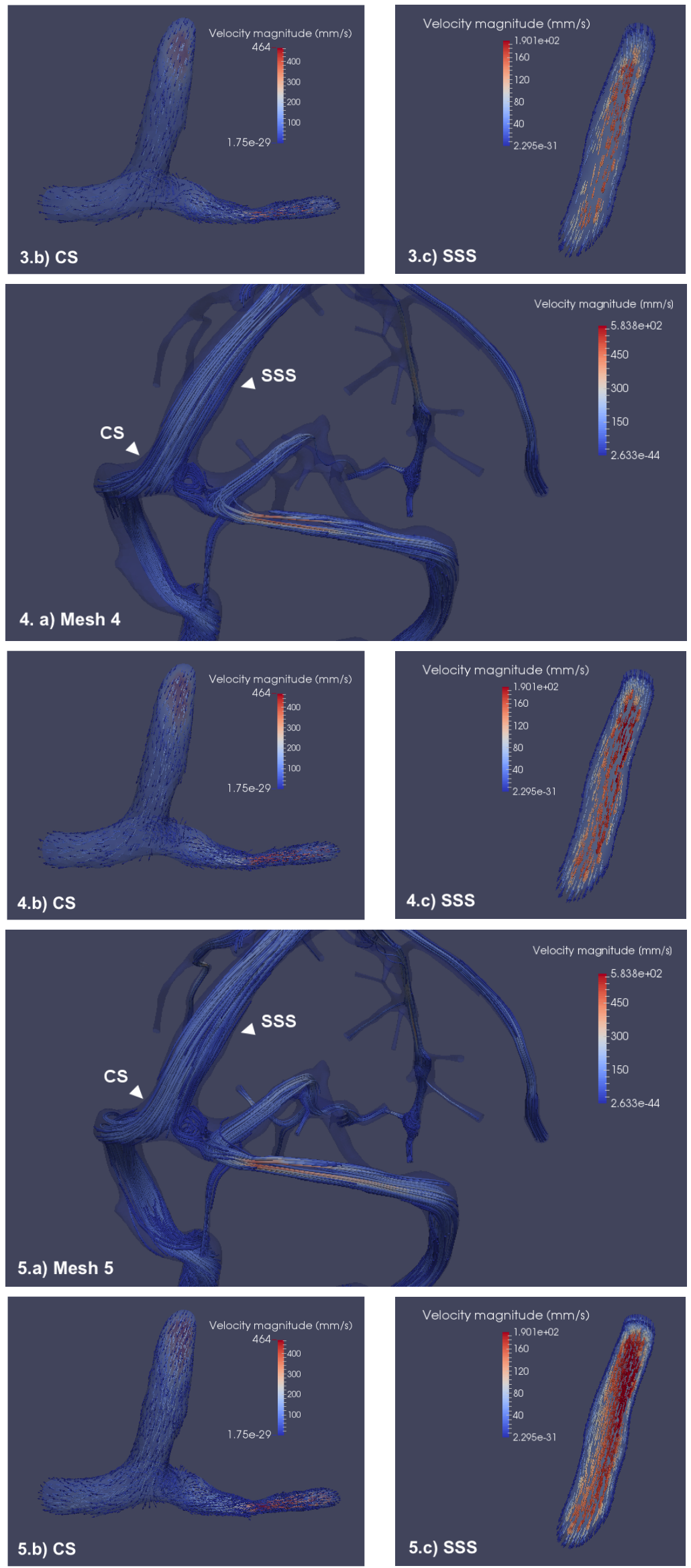

Figure 2. Unsteady Navier-Stokes simulation (iteration 135 over 200, i.e. 0,35 second in the second cardiac cycle): on the top N.a), the streamlines of the whole cerebral venous network, below on the left N.b), the velocity field on the confluence of sinuses and on the right N.c), the velocity field on the superior sagittal sinus (vectors represent the velocity direction and magnitude and colors represent the Y velocity component). Each Nth set a), b) and c) corresponds to the Nth mesh. 


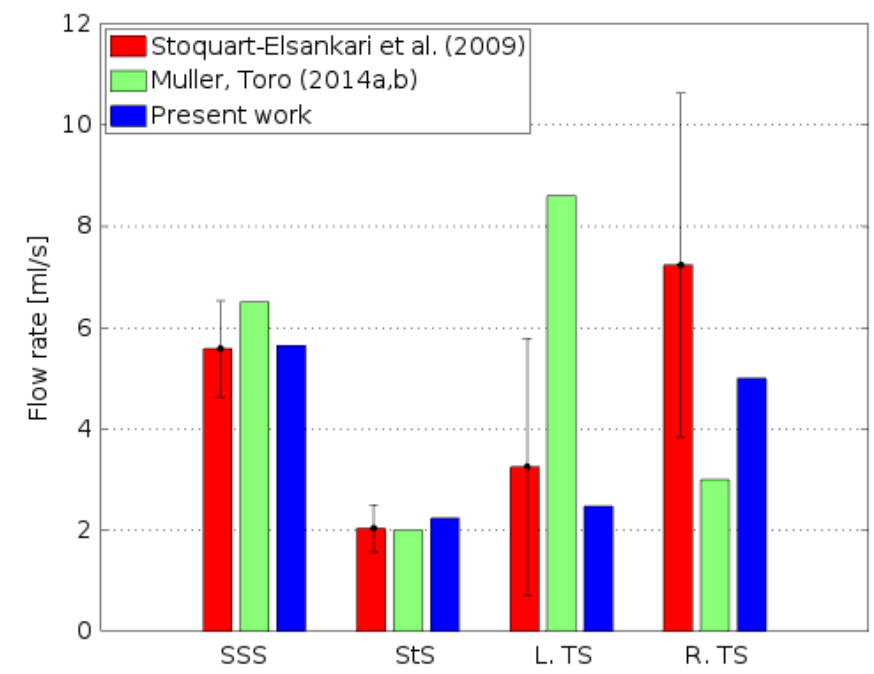

Figure 3. Comparison between MRI measurements of flow rates $(\mathrm{ml} / \mathrm{s}$ )(average and standard deviation) found in literature and our model in different locations of the intracranial venous network. SSS : Superior Sagittal Sinus Fig. 1 (2), St. S : Straight Sinus Fig. 1 (3), L. TS: Left Transverse Sinus Fig. 1 (5), R. TS: Right Transverse Sinus Fig. 1 (5)

current 1D models to take into account the helical motion induced by the vessel curvature. In the intracranial arterial network, a good agreement between both models can be obtained when arterial wall elasticity is ignored (Grinberg et al. (2011)). However, fluctuations in the pressure and flow rates predicted by the rigid wall model are significantly higher than those predicted by the flexible wall model.

The present investigation provides information on blood flow behaviour using a three-dimensional personalized geometry. The 3D computational model showed that a jet can be observed during the beginning of the decelerating phase after peak flow in the entrance segment of the right transverse sinus downstream from a slight constriction, probably due to the printing of an adjoining anatomical element in this healthy young volunteer or a reconstruction artifact. This fact highlights the advantage of a complete process that starts from acquired image processing for 3D reconstruction of the vasculature of interest to reconstruction of virtual images. Then, by comparison between acquired and reconstructed images, one can assess the cause of any constriction along the vascular circuit and hence eliminate or confirm an artifact.

\subsection{Newtonian assumption}

A Newtonian model was adopted for blood rheology. In addition to the arguments developed in Section 3, blood flow simulations in cerebral aneurysms were not sensitive to blood rheology models (Gambaruto et al. (2011)). Moreover, the blood viscosity model influences to a lesser extent blood flow computations than the modeling assumptions on the boundary conditions, especially inlet conditions, except when a precise computation of the wall shear stress is needed (Chabannes et al. (2015)).

\subsection{Rigid walls}

A relevant model describing the deformability of the cerebral venous wall remains a challenging issue for several reasons: (i) in vivo assessments of material properties are not perfectly handled; (ii) midsize vessels are not accessible to ultra-sound elastography for estimation of rheological parameters; 
(iii) in vitro measurements in samples collected from dead individuals are accurately managed, but not performed in perfused living tissues, and (iv) the state of the art in fluid-structure interaction does not take into account the multilayered wall made up of composite materials. Therefore, even in recent $3 \mathrm{D}$ simulations of cerebrovenous flows such as those in jugular veins, walls are also assumed to be rigid (Caiazzo et al. (2015)).

\subsection{Boundary conditions}

This contribution is a first step towards multiscale models, in which 3D simulations are carried out in the region of interest and are coupled with reduced models of other compartments of the cardiovascular system, including the cardiac pump. To the best of our knowledge, only very few works are available in the case of the venous network (3D simulations of blood flow in the jugular veins coupled with a 1D model in major intracranial veins (Caiazzo et al. (2015)) and coupling of a 3D model with a simplified three-elements Windkessel model (Chabannes et al. (2015)), which represent in fact partial modellings. Moreover, the mechanical parameters are, most often, not obtained in the same individuals.

The extracranial compartment of the cerebral venous drainage is more deformable than the intracranial one (Stoquart-Elsankari et al. (2009)). Cervical veins such as the upstream segment of the internal jugular vein that runs vertically down the neck side close to the internal carotid artery undergo significant deformations due to pressure pulses propagating through the carotid artery. These transient strains are here neglected because the present study focuses on the intracranial compartment, the virtual exit veins serving only for proper application of outlet boundary conditions.

Artificial inflow and outflow boundaries are introduced by cutting the computational domain from the rest of the circulatory loop. The inflow velocity of a venous circuit has a quasi-steady profile, a sensitivity analysis of its magnitude being performed in (Chabannes et al. (2015)). The results showed that for cerebral veins, setting the inlet boundary condition has an important impact on the forces created by blood flow, thus highlighting the importance of deriving values for these conditions from clinically measured data at some probe stations in order to enhance the accuracy of the computed hemodynamical quantities of interest. However, technical limitations do not allow these measurements in small-bore vessels such as entry veins of the present model.

Simple coupling of the downstream vasculature of the neck and cranial part of the thorax using reduced models relies not only on electrical analogues (0D models), which yet should be used with proper estimation of personalised parameter values, but also adequate 1D model of deformable (both distensible and collapsible) veins to avoid reflection artifacts of pressure waves. Hence, a proper personalised vein law must be first determined. Furthermore, the unsteady venous outflow depends on the cyclic changes in internal and external pressures associated with the venous return in the right atrium of the beating heart under the influence of breathing.

The body's position yields another constraint, as it affects the outlet drainage path and hence flow distribution among all possible exit vessels (Gisolf et al. (2004)). Therefore, the present computational domain focuses on the intracranial compartment.

\subsection{Computational domain}

The computational domain must derive from a relevant geometry, in addition to an accurate topology. Slight errors in the geometric modeling can lead to erroneous blood flow distribution 


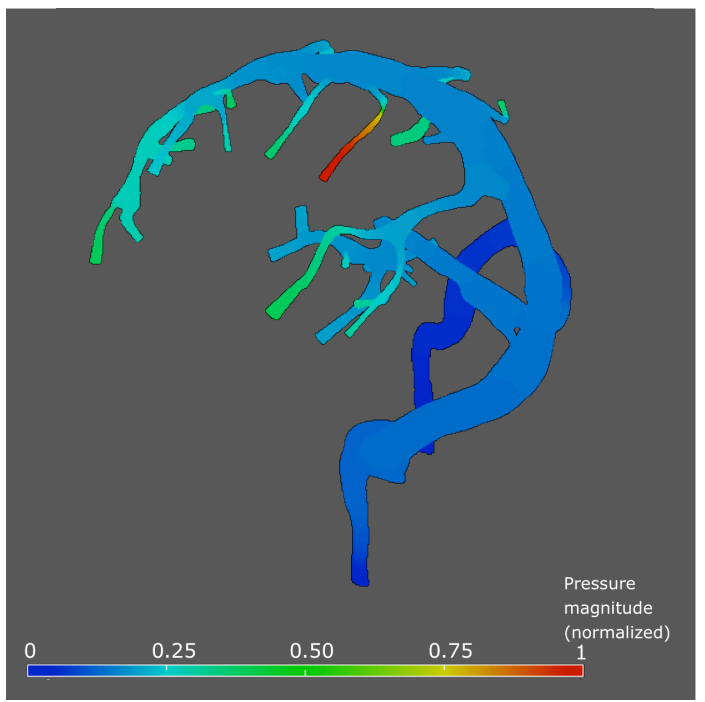

Figure 4. Side effects of geometric modeling inaccuracies on blood flow simulation relevance. A local vessel diameter reduction can lead to an abnormally elevated pressure field (normalized values maximum in red).

(Figure 4), the global computed pressure field being affected by a local geometry alteration that can result from anatomical influences or reconstruction artefacts. Comparison of original images to reconstructed ones will allow us to analyze the cause of any constriction along the vascular circuit and hence eliminate or confirm an artifact.

\section{Concluding remarks}

A methodology was presented for subject-specific blood flow simulations in the intracranial venous network to prove the feasibility of the pipeline from medical image acquisition to simulation. A new algorithm is currently under development and it will be used to carry out high-performance computing that allows the use of refined meshes. In addition, both anatomical and functional imaging (i.e., associated with MR velocimetry) is beginning to investigate 3D blood flow simulation in macrovascular networks.

One of the main open questions is linked to the validation of the results using phantoms or, better, in vivo measurements by functional imaging. However, measurement themselves have limitations due to space and time resolution of devices and vessel accessibility to measurements. The development of virtual angiography, which simulates the physical image acquisition sequences to create realistic synthetic data, provides a promising perspective that helps to interpret some features of the derived computational meshes (Fortin et al. (2014); Ford et al. (2005); Jurczuk et al. (2012)).

\section{References}

Boissonnat JD, Chaine R, Frey P, Malandain G, Salmon S, Saltel E, Thiriet M. From arteriographies to computational flow in saccular aneurisms: The INRIA experience. Medical Image Analysis 2005; 9:133143.

Caiazzo A, Montecinos G, Müller LO, Toro EF, Haacke EM. Computational haemodynamics in stenotic internal jugular veins. Journal of Mathematical Biology 2015; 70, 745-772.

Cebral JR, Castro MA, Appanaboyina S, Putman CM, Millan D, Frangi AF. Efficient pipeline for imagebased patient-specific analysis of cerebral aneurysm hemodynamics: Technique and sensitivity. IEEE Transactions on Medical Imaging 2005; 24:457-467. 
Chabannes V, Ismail M, Prud'Homme C, Szopos M. Hemodynamic simulations in the cerebral venous network: A study on the influence of different modeling assumptions. Journal of Coupled Systems and Multiscale Dynamics 2015; 3:23-37.

Corsini C, Baker C, Kung E, Schievano S, Arbia G, Baretta A, Dorfman A. An integrated approach to patient-specific predictive modeling for single ventricle heart palliation. Computer Methods in Biomechanics and Biomedical Engineering 2014;17:1572-1589.

Ford MD, Stuhne GR, Nikolov HN, Habets DF, Lownie SP, Holdsworth DW, Steinman DA. Virtual angiography for visualization and validation of computational models of aneurysm hemodynamics. IEEE Transactions on Medical Imaging 2005; 24:1586-1592.

Formaggia L, Perktold K, Quarteroni A. Basic mathematical models and motivations. In Cardiovascular Mathematics. Modeling and Simulation of the Circulatory System, Formaggia L, Quarteroni A, Veneziani A (eds). Springer, MS\&A 1; 2009.

Fortin A, Durand E, Salmon S. Extension of an MRI simulator software for phase contrast angiography experiments. International Symposium on Biomedical Simulation (ISBMS), Lecture Notes in Computer Science, Springer 2014; 8789:150-154.

Gadda G, Taibi A, Sisini F, Gambaccini M, Zamboni P, Ursino M. A new hemodynamic model for the study of cerebral venous outflow. American Journal of Physiology - Heart and Circulatory Physiology, 2015; 308:H217-H231.

Gambaruto A, Janela J, Moura A, Sequeira A. Sensitivity of haemodynamics in patient specific cerebral aneurysms to vascular geometry and blood rheology. Mathematical Biosciences and Engineering 2011; 8:409-423.

Gideon, P., Thomsen, C., Gjerris, F., Sørensen, P. S., Ståhlberg, F., Henriksen, O. Measurement of blood flow in the superior sagittal sinus in healthy volunteers, and in patients with normal pressure hydrocephalus and idiopathic intracranial hypertension with phase-contrast cine MR imaging. Acta Radiologica 1996;37(1P1):171-176.

Girault V, Raviart PA. Finite Element Methods for Navier-Stokes Equations. Springer-Verlag, 1986.

Gisolf J, van Lieshout JJ, van Heusden K, Pott F, Stok WJ, Karemaker JM. Human cerebral venous outflow pathway depends on posture and central venous pressure. Journal of Physiology 2004; 560:317-327.

Grinberg L, Cheever E, Anor T, Madsen JR, Karniadakis GE. Modeling blood flow circulation in intracranial arterial networks: A comparative 3D/1D simulation study. Ann Biomed Eng 2011; 39:297-309.

Hecht F. New development in Freefem++. Journal of Numerical Mathematics 2012; 20:251-265.

Ho H, Mithraratne K, Hunter P. Numerical simulation of blood flow in an anatomically-accurate cerebral venous tree. IEEE Transactions on Medical Imaging 2013; 32:85-91.

Jurczuk K, Kretowski M, Eliat P-A, Bellanger J-J, Saint-Jalmes H, Bézy-Wendling J. A new approach in combined modeling of MRI and blood flow: A preliminary study, In International Symposium on Biomedical Imaging 2012; 812-815.

Kiliç, T and Akakin, A. Anatomy of cerebral veins and sinuses. Front Neurol Neurosci. 2008; 23:4-15.

Miraucourt O, Garnotel S. Numerical Tour of Fluid Dynamics. http://numtourcfd.univ-reims.fr.

Miraucourt O, Génevaux O, Szopos M, Thiriet M, Talbot H, Salmon S, Passat N. 3D CFD in complex vascular systems: A case study. International Symposium on Biomedical Simulation (ISBMS), Lecture Notes in Computer Science, Springer 2014; 8789:86-94.

Miyazaki M, Lee VS. Nonenhanced MR angiography. Radiology 2008; 248:20-43.

Morales HG, Larrabide I, Geers AJ, San Román L, Blasco J, Macho JM, Frangi AF. A virtual coiling technique for image-based aneurysm models by dynamic path planning. IEEE Transactions on Medical Imaging 2013; 32:119-129.

Müller LO, Toro EF. A global multiscale mathematical model for the human circulation with emphasis on the venous system. International Journal for Numerical Methods in Biomedical Engineering 2014; 30:681-725.

Müller LO, Toro EF. Enhanced global mathematical model for studying cerebral venous blood flow. Journal of biomechanics, 2014; 47:3361-3372.

Ogoh S, Sato K, Fisher JP, Seifert T, Overgaard M, Secher NH. The effect of phenylephrine on arterial and venous cerebral blood flow in healthy subjects. Clinical Physiology and Functional Imaging 2011; 31:445-451.

Ozsarlak O, Van Goethem JW, Maes M, Parizel PM. MR angiography of the intracranial vessels: Technical aspects and clinical applications. Neuroradiology 2004; 46:955-972.

Passerini T, De Luca M, Formaggia L, Quarteroni A, Veneziani A. A 3D/1D geometrical multiscale model 
of cerebral vasculature. Journal of Engineering Mathematics 2009; 64:319-330.

Pironneau O. On the transport-diffusion algorithm and its applications to the Navier-Stokes equations. Numerische Mathematik 1982; 38:309-332.

Retarekar R, Ramachandran M, Berkowitz B, Harbaugh RE, Hasan D, Rosenwasser RH, Ogilvy CS, Raghavan ML. Stratification of a population of intracranial aneurysms using blood flow metrics. Computer Methods in Biomechanics and Biomedical Engineering, 2015; 18:1072-1082.

Reymond P, Merenda F, Perren F, Rüfenacht D, Stergiopulos N. Validation of a one-dimensional model of the systemic arterial tree. American Journal of Physiology - Heart and Circulatory Physiology 2009; 297:208-222.

Schaller B. Physiology of cerebral venous blood flow: From experimental data in animals to normal function in humans. Brain Research. Brain Research Reviews 2004; 46:243-260.

Sforza DM, Löhner R, Putman C, Cebral JR. Hemodynamic analysis of intracranial aneurysms with moving parent arteries: Basilar tip aneurysms. International Journal for Numerical Methods in Biomedical Engineering 2010; 26:1219-1227.

Shojima M, Morita A, Kimura T, Oshima M, Kin T, Saito N. Computational fluid dynamic simulation of a giant basilar tip aneurysm with eventual rupture after hunterian ligation. World neurosurgery 2014; 82:535.e5-535.e9.

Stoquart-Elsankari S, Lehmann P, Villette A, Czosnyka M, Meyer ME, Deramond H, Balédent O. A phasecontrast MRI study of physiologic cerebral venous flow. Journal of Cerebral Blood Flow and Metabolism 2009; 29:1208-1215.

Thiriet M. Biology and Mechanics of Blood Flows, part I: Biology of Blood Flows, part II: Mechanics and Medical Aspects of Blood Flows. Springer, CRM Series in Mathematical Physics, 2008.

Thiriet M. Cell and Tissue Organization in the Circulatory and Ventilatory Systems. Springer, Biomathematical and Biomechanical Modeling of the Circulatory and Ventilatory Systems 1; 2011.

Ursino M, Lodi CA. A simple mathematical model of the interaction between intracranial pressure and cerebral hemodynamics. Journal of Applied Physiology, 1997; 82:1256-1269.

Wang S, Ding G, Zhang Y, Yang X. Computational haemodynamics in two idealised cerebral wide-necked aneurysms after stent placement. Computer Methods in Biomechanics and Biomedical Engineering 2011; 14:927-937.

Xiao N, Humphrey JD, Figueroa CA. Multi-scale computational model of three-dimensional hemodynamics within a deformable full-body arterial network. Journal of Computational Physics 2013; 244:22-40.

Xiao N, Alastruey J, Figueroa CA. A systematic comparison between 1-D and 3-D hemodynamics in compliant arterial models. International Journal for Numerical Methods in Biomedical Engineering 2014; 30:204-231. 